\title{
Comparison of Misalignment and Retardation Errors of Dual Rotating Quarter-Wave Plates in Muller-Matrix Ellipsometry
}

\author{
Hai Du Cheong ${ }^{\dagger}$ and Dukhyeon Kim \\ Division of Liberal Arts, Hanbat National University, Daejeon 305-719, Korea
}

(Received August 22, 2014; Revised manuscript September 25, 2014; Accepted September 26, 2014)

\begin{abstract}
Using an ellipsometer with dual rotating quarter-wave plates, we have analyzed the relationship between Fourier coefficients and Mueller matrices in the cases of an error-free optical system and of five systematic errors (alignment errors and retardation errors in the quarter-wave plates, and alignment error in the analyzer). In the case with five systematic errors, simulation results show that retardation errors cause more error in the diagonal elements of the Mueller matrix than do alignment errors. We have found that errors in the Mueller matrix caused by initial misalignment of the dual quarter-wave plates were the same. We have chosen the rotation rates of two quarter-wave plates such that the rotational frequencies $\omega_{1}$ and $\omega_{2}$ differ by a factor of 5 , i.e. $\omega_{2}=5 \omega_{1}$. The simulation results show $0.18 \%$ relative error in the diagonal elements $\left(m_{22}\right.$ and $\left.m_{33}\right)$ and $200 \%$ relative error in the off-diagonal elements $\left(m_{23}\right.$ and $m_{32}$ ), when we compare errors caused by misalignment of the analyzer to those caused by initial misalignment of the quarter-wave plates. We can use these results in measuring accurate Mueller matrices of optical materials.
\end{abstract}

Keywords: Ellipsometry, Dual rotating ellipsometry, Alignment error, Retardation error, Mueller matrix

OCIS codes: (120.2130) Ellipsometry and polarimetry; (120.4640) Optical instruments; (000.2170) Equipment and techniques

\section{타원편광 분석기에서 정렬오차와 사분파장판의 지연오차로 인한 뮬러 매트릭스 오차 분석 \\ 정해두 ${ }^{\dagger}$. 김덕현 \\ 한밭대학교 교양학부 \\ (ㅇ) 305-719 대전광역시 유성구 동서대로 125 번지}

(2014년 8월 22일 받음, 2014년 9월 25일 수정본 받음, 2014년 9월 26일 게재 확정)

두 개의 회전하는 사분파장판을 가진 타원편광분석기에서 광학계가 오차를 포함하지 않는 경우와 5 가지의 체계적 오차(두 개 의 사분파장판의 정렬오차와 지연오차, 검광판의 정렬오차)를 포함하는 경우에 측정신호를 핏팅하여 구한 퓨리어 계수와 뮬러 매트릭스와의 관계를 분석하였다. 5 가지 체계적 오차를 포함하는 경우 전산모사를 이용해 비교 분석한 결과 사분파장판의 지연 오차가 정렬오차보다 뮬러매트릭스 대각선 요소에 야기하는 오차가 더 크다는 것을 찾아내었다. 각속도의 회전비를 1:5로 선택시 첫 번째 사분파장판의 초기 정렬오차와 두 번째 사분 파장판의 초기 정렬오차가 뮬러 매트릭스에 야기하는 오차가 같다는 것을 전모사를 통해 찾아 내었다. 5 가지 체계적인 오차를 전산모사를 통해 분석한 결과 검광판의 정렬오차 $\left(\epsilon_{5}\right)$ 가 야기하는 뮬러 매트 릭스 오차와 사분파장판의 정렬오차 $\left(\epsilon_{3}, \epsilon_{4}\right)$ 가 야기하는 뮬러 매트릭스 오차를 비교하면 대각선 요소 $\left(m_{22}\right.$ 와 $\left.m_{33}\right)$ 의 상대 오차는 $0.18 \%$ 이며 비 대각선요소 $\left(m_{23}\right.$ 와 $\left.m_{32}\right)$ 는 2 배 차이가 남을 찾아내었다. 이 결과들을 활용하여 측정대상 물질의 보다 정확한 뮬러 매트릭스를 얻을 수 있다.

Keywords: 타원편광분석기, 회전형 편광분석기, 정렬오차, 지연오차, 뮬러 매트릭스

OCIS codes: (120.2130) Ellipsometry and polarimetry; (120.4640) Optical instruments; (000.2170) Equipment and techniques

${ }^{\dagger}$ E-mail: hdcheong@hanbat.ac.kr 


\section{I. 서 론}

물질의 광학적 특성을 측정하는 데 시편 앞에 놓인 편광판 을 회전 시키는 타원편광분석기, 시편 뒤에 놓인 검광판을 회전 시키는 타원 편광 분석기, 시편 앞, 혹은 뒤에 놓인 사 분파장판을 회전 시키는 타원편광 분석기 등의 다양한 방법 [1-6, 10-15]이 사용되고 있는 데 본 연구에서는 시편 앞과 시편 뒤에 놓인 두 개의 사분파장판을 회전 시키는 타원 편광분석 기 ${ }^{[7-9]}$ 를 사용한다. 두 개의 사분파장판의 각속도 비를 다양 하게 선택할 수 있지만 회전 각속도의 비를 1:5로 정하여 측 정한 신호를 이용하여 계산한 측정 물질의 뮬러 매트릭스가 가장 안정적이다. 실험시 타원편광분석기를 구성하는 각 광 학계(사분파장판, 검광판)의 정렬오차와 사분파장판의 지연 오차로 인하여 야기되는 문제로 측정물질의 정확한 뮬러 매 트릭스를 얻기가 어렵다. 본 연구에 사용된 타원편광분석기 에서 5 가지 종류의 체계적인 오차가 뮬러 매트릭스에 얼마 만큼의 오차를 야기 시키는 지를 전산모사를 통해 비교 분석 하였다. 5 가지의 체계적인 오차는 첫 번째 사분파장판의 초 기 각도 정렬오차와 지연오차 $\left(\Delta \theta_{1}, \Delta \delta_{1}\right)$, 두 번째 사분파장 판의 초기 각도 정렬 오차와 지연오차 $\left(\Delta \theta_{2}, \Delta \delta_{2}\right)$, 검광판 (analyzer)의 투과축 각도와 편광판(polarizer)의 투과축 각도 와의 차이 $\left(\Delta \theta_{3}\right)$ 이다.

\section{II. 기본이론 및 타원편광 분석기 구성}

본 연구에서 사용한 타원편광분석기는 두 개의 편광판(편광 판과 검광판)과 회전하는 두 개의 사분 파장판으로 구성된다.

타원편광분석기의 구조는 Fig.1과 같으며 각 광학계를 뮬 러 매트릭스로 나타내고 광 검출기를 통하여 얻게 되는 강도 신호는 식 (1)과 같다.

$$
\begin{aligned}
I\left(\delta_{1}, \theta_{1}, \delta_{2}, \theta_{2}, \theta_{3}\right) & =[c, 0,0,0] P L_{2}\left(\theta_{3}\right) W P_{2}\left(\delta_{2}, \theta_{2}\right) \\
& -M W P_{1}\left(\delta_{1}, \theta_{1}\right) P L_{1}[1,0,0,0]^{T}
\end{aligned}
$$

여기서 $\delta_{1}, \theta_{1}, \delta_{2}, \theta_{2}, \theta_{3}$ 는 각각 광원에서부터, 첫 번째 회전하

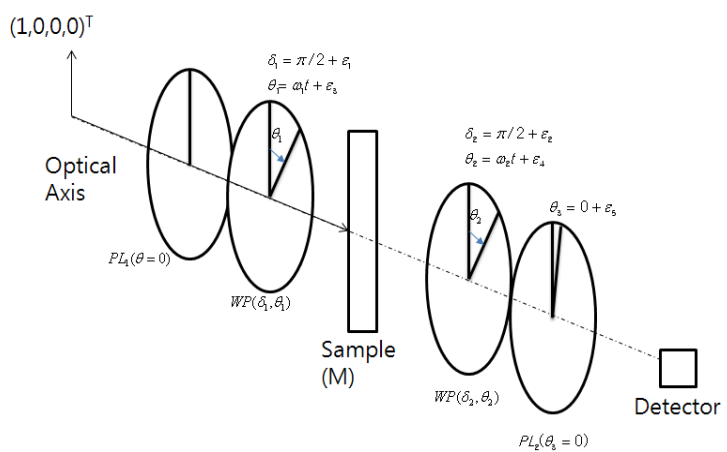

FIG. 1. Baisc configuration of the Muller matrix ellipsometry system with two rotating quarter-wave plates.
는 사분파장판 $\left(W P_{1}\right)$ 의 빠른 축(fast axis)과 느린 축(slow axis)간의 빛의 지연오차, 빠른 축과 편광판 $\left(P L_{1}\right)$ 의 투과축 이 이루는 각도, 두 번째 사분파장판의 지연오차 및 각도, 검 출기 앞에 설치한 검광판 $\left(P L_{2}\right)$ 의 투과축과 편광판 $\left(P L_{1}\right)$ 의 투과축이 이루는 각도, $\mathrm{M}$ 은 측정대상 광학계(측정대상 물질) 의 뮬러 매트릭스를 나타내며 $[c, 0,0,0]$ 는 빛의 강도를 측정 하는 검출기의 효율(c)을 포함한 벡터, $[1,0,0,0]^{T}$ 는 편광된 정규화된 입력빔의 스토크 벡터(Stokes vector)를 나타낸다. 식(1)을 측정하고자 하는 물질(M)의 앞부분에 있는 모든 광 학계와 뒷부분에 있는 광학계를 나누어 표현하면 식 (2)와 같다.

$$
\begin{aligned}
I\left(\delta_{1}, \theta_{1}, \delta_{2}, \theta_{2}, \theta_{3}\right)= & {[c, 0,0,0] P L_{2}\left(\theta_{3}\right) W P_{2}\left(\delta_{2}, \theta_{2}\right) } \\
\bullet & W P_{1}\left(\delta_{1}, \theta_{1}\right) P L_{1}(\theta=0)[1,0,0,0]^{T} \\
& =c A \cdot M \cdot P \\
& =c \sum_{i=1}^{4} \sum_{j=1}^{4} A_{i} M_{i j} P_{j}
\end{aligned}
$$

여기서 벡터P와 $\mathrm{A}$ 는 각각 측정하고자 하는 물질(M)의 앞부 분, 뒷부분에 있는 광학계의 모든 것을 포함하여 다음과 같 은 벡터로 간단히 나타낸다.

$$
\begin{aligned}
& P=\left[p_{1}, p_{2}, p_{3}, p_{4}\right]^{T} \\
& A=\left[a_{1}, a_{2}, a_{3}, a_{4}\right]
\end{aligned}
$$

여기서 T(transpose)는 행렬의 자리바꿈을 나타낸다.

처음 시각 $(\mathrm{t}=0)$ 에서 두 개의 사분파장판의 빠른 축과 편광 판과 검광판의 투과축은 같은 방향으로 정렬되어 있으면 측 정 신호 해석이 용이하다. 사분 파장판의 빠른 축이 편광판 의 투과축 방향과 일치하지 않고 조금 차이가 있게 회전되어 있는 경우 $\left(\theta_{i}\right)$, 회전된 사분파장판(WP)의 뮬러 매트릭스는 다음 식(5)와 같이 표현된다.

$$
W P\left(\theta_{i}\right)=R\left(-\theta_{i}\right)\left[\begin{array}{cccc}
1 & 0 & 0 & 0 \\
0 & 1 & 0 & 0 \\
0 & 0 & \cos \left(\delta_{i}\right) & \sin \left(\delta_{i}\right) \\
0 & 0 & -\sin \left(\delta_{i}\right) & \cos \left(\delta_{i}\right)
\end{array}\right] R\left(\theta_{i}\right)
$$

여기서, $\delta_{i}$ 는 사분파장판의 빠른 축과 느린 축과의 위상차, 각도 $\theta_{i}$ 는 빠른 축이 편광판의 투과축 방향에 대해 회전한 각도를 나타낸다. 그리고 $R(\theta)$ 의 매트릭스는 아래식(6)과 같다.

$$
R(\theta)=\left[\begin{array}{cccc}
1 & 0 & 0 & 0 \\
0 & \cos (2 \theta) & \sin (2 \theta) & 0 \\
0-\sin (2 \theta) & \cos (2 \theta) & 0 \\
0 & 0 & 0 & 1
\end{array}\right]
$$




\subsection{5 가지 체계적인 오차가 포함되지 않은 경우}

두 개의 사분파장판을 $1: 5\left(\omega_{1}=\omega, \omega_{2}=5 \omega\right)$ 의 각속도 회전 비로 회전 시키는 경우를 분석한다. 5가지의 체계적인 오차 를 포함하지 않는 두 개의 사분파장판이 회전하는 타원편광 분석기의 시간적인 강도신호는 식 (7)로 표현된다.

$$
\begin{aligned}
I\left(\theta_{1}, \theta_{2}\right) & =I(\omega t) \\
& =c\left\{a_{0}+\sum_{j=1}^{12}\left[a_{j} \cos (2 j \omega t)+b_{j} \sin (2 j \omega t)\right]\right\}
\end{aligned}
$$

여기서, $a_{j}, b_{j}$ 는 측정하고자 하는 물질(광학계)의 뮬러 매트 릭스 구성요소와 관계가 있다. 이 때 식(7)을 12 가지 종류의 주기를 가진 사인 및 코사인 함수들로 핏팅(fitting)하여 그 퓨리어 계수 $\left(a_{j}, b\right)$ 를 구하여 측정하고자 하는 광학계의 뮬러 매트릭스를 계산해 낸다.

퓨리어 계수 $\left(a_{0}, a_{1}, \cdots \cdot a_{12}, b_{1}, \cdots \cdot b_{12}\right)$ 를 구하는 것은 식(7)과 같이 주어진 일정한 간격으로 표현되는 여러 가지 주기함수의 진폭을 구하는 것과 같다. 12 가지 종류의 주기를 가진 사인 및 코사인 함수의 진폭을 구하기 위해 식(7)을 아 래식과 같이 다시 나타낸다.

$$
\begin{aligned}
& X=\left(a_{0}, a_{1}, \bullet \bullet a_{12}, b_{1}, \bullet \cdot b_{12}\right)^{T} \\
& Y=\left(I\left(t_{1}\right), I\left(t_{2}\right), I\left(t_{3}\right), \bullet \bullet \quad I\left(t_{N}\right)\right)^{T}
\end{aligned}
$$

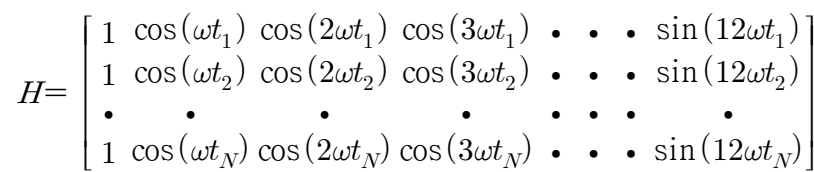

$$
Y=H X
$$

여기서, $X, Y, H$ 는 각각 구하고자 하는 해 벡터, 측정 신호 벡터, 12 가지 종류의 사인 및 코사인 주기함수로 구성된 매 트릭스를 나타낸다.

여기서, 측정 신호 벡터(Y)에 있는 $t_{1}, \cdots \cdot t_{N}$ 은 광 검출 기의 출력을 디지털 신호로 바꾸어 주는 $\mathrm{ADC}$ (Analogue Digital Converter)의 샘플링(sampling)시간을 각각 나타내며 식 (11)을 만족하는 해 벡터는 아래식을 이용해 계산할 수 있다.

$$
X=\left(H^{T} H\right)^{-1} H^{T} Y
$$

식 (7)을 선형 핏팅 계산으로 구하는 25 개의 퓨리어 계수 $\left(a_{0}, a_{1}, \cdots-a_{12}, b_{1}, \cdots \bullet b_{12}\right)$ 와 구하고자 하는 16 개의 뮬 러 매트릭스 구성요소는 서로 선형적 관계가 있으며 과 평가 된(overestimated) 선형 연립방정식의 풀이 문제로 귀착된다. 5 가지 체계적인 오차를 포함하지 않는 경우에 측정 신호를 퓨리어 변환을 통해 구한 퓨리어 계수와 측정대상 물질(광학 계)의 뮬러 매트릭스 구성요소와 관계는 식 (13)과 같다.

$$
\begin{aligned}
& a_{0}=m_{11}+\frac{1}{2} m_{12} \\
& a_{2}=\frac{1}{2} m_{12}+\frac{1}{4} m_{22} \\
& a_{3}=-\frac{1}{4} m_{43} \\
& a_{4}=-\frac{1}{2} m_{44} \\
& a_{6}=\frac{1}{2} m_{44} \\
& a_{7}=\frac{1}{4} m_{43} \\
& a_{8}=\frac{1}{8} m_{22}+\frac{1}{8} m_{33} \\
& a_{9}=\frac{1}{4} m_{34} \\
& a_{10}=\frac{1}{2} m_{21}+\frac{1}{4} m_{22} \\
& a_{11}=-\frac{1}{4} m_{34} \\
& a_{12}=\frac{1}{8} m_{22}-\frac{1}{8} m_{33} \\
& a_{1}=a_{5}=0 \\
& b_{1}=m_{14}+\frac{1}{2} m_{24} \\
& b_{2}=\frac{1}{2} m_{13}+\frac{1}{4} m_{23} \\
& b_{3}=-\frac{1}{4} m_{42} \\
& b_{5}=-m_{41}-\frac{1}{2} m_{42} \\
& b_{7}=-\frac{1}{4} m_{42} \\
& b_{8}=-\frac{1}{8} m_{23}+\frac{1}{8} m_{32} \\
& b_{9}=-\frac{1}{4} m_{24} \\
& b_{10}=\frac{1}{2} m_{31}+\frac{1}{4} m_{32} \\
& b_{11}=\frac{1}{4} m_{24} \\
& b_{12}=\frac{1}{8} m_{23}+\frac{1}{8} m_{32} \\
& b_{4}=b_{6}=0
\end{aligned}
$$


위의 관계식을 이용하여 뮬러 매트릭스 구성요소를 구한 결과는 식 (14)와 같다.

$$
\begin{aligned}
& m_{11}=a_{0}-a_{2}+a_{8}-a_{10}+a_{12} \\
& m_{12}=8 a_{2}-8 a_{8}-8 a_{12} \\
& m_{13}=8 b_{2}+8 b_{8}-8 b_{12} \\
& m_{14}=4 b_{1}-8 b_{11}=4 b_{1}+8 b_{9}=4 b_{1}+4 b_{9}-4 b_{11} \\
& m_{21}=-8 a_{8}+8 a_{10}-8 a_{12} \\
& m_{22}=16 a_{8}+16 a_{12} \\
& m_{23}=-16 b_{8}+16 b_{12} \\
& m_{24}=-16 b_{9}=16 b_{11}=8\left(-b_{9}+b_{11}\right) \\
& m_{31}=-8 b_{8}+8 b_{10}-8 b_{12} \\
& m_{32}=16 b_{8}+16 b_{12} \\
& m_{33}=16 a_{8}-16 a_{12} \\
& m_{34}=16 a_{9}=-16 a_{11}=8\left(a_{9}-a_{11}\right) \\
& m_{41}=8 b_{3}-4 b_{5}=-4 b_{5}+8 b_{7}=4\left(b_{3}-b_{5}+b_{7}\right) \\
& m_{42}=-16 b_{3}=-16 b_{7}=-8\left(b_{3}+b_{7}\right) \\
& m_{43}=-16 a_{3}=16 a_{7}=8\left(-a_{3}+a_{7}\right) \\
& m_{44}=-8 a_{4}=8 a_{6}=4\left(a_{6}-a_{4}\right)
\end{aligned}
$$

\subsection{5 가지의 체계적 오차 $\left(\delta_{1}, \theta_{1}, \delta_{2}, \theta_{2}, \theta_{3}\right)$ 를 고려한 경우}

사분파장판의 두께가 온도에 따라 변하게 되거나, 사분파 장판이 광축에 대하여 $90^{0}$ 와 차이가 있을 경우 광 경로차가 $\pi / 2$ 와 차이가 발생하며 또한 처음 시각 $(\mathrm{t}=0)$ 에서 사분 파장 판의 빠른 축과 첫 번째 편광판의 투과축 방향이 일치하지 않을 수 있고, 마찬가지로 두 번째 검광판(analyzer)의 투과 축 각도가 첫 번째 편광판(polarizer)의 방향과 일치하지 않을 경우 각각의 물리량은 오차를 포함하게 되며 식 (20)으로 나 타내어진다.

$$
\begin{aligned}
& \delta_{1}=\pi / 2+\varepsilon_{1}=\delta_{1}^{0}+\varepsilon_{1} \\
& \delta_{2}=\pi / 2+\varepsilon_{2}=\delta_{2}^{0}+\varepsilon_{2}
\end{aligned}
$$

$$
\begin{aligned}
& \theta_{1}(t)=\omega t+\varepsilon_{3} \\
& \theta_{2}(t)=5 \omega t+\varepsilon_{4} \\
& \theta_{3}(t)=0+\varepsilon_{5}
\end{aligned}
$$

식 (18)에서 Goldstein ${ }^{[3]}$ 등이 고려한 오차는 $\theta_{2}(t)=5\left(\omega+\varepsilon_{4}\right) t$ 이나, 본 연구에서는 일반적으로 식 (15)와 식 (16)과 같이 표현되는 초기 각도 오차(initial offset angle)가 실험시 피할 수 없는 물리량이 되고 매 실험시 다를 수 있으며, 이 초기 각도 오차 보정이 Goldstein ${ }^{[3]}$ 의 각속도 오차보정 보다 더 중요하다고 판단되어 이 오차 보정에 중점을 두어 분석한 결 과는 논문 ${ }^{[15]}$ 에 실었다. 또한 두 번째 검광판(analyzer)이 첫 번째 편광판(polarizer)의 방향이 일치 하지 않을 경우 식 (19) 에 나타난 것 같이 오차 $\left(\varepsilon_{5}\right)$ 를 포함하게 되어 검광판 $\left(P L_{2}\right)$ 의 뮬러 매트릭스는 식 (20)으로 나타내어진다.

$$
P L_{2}\left(\theta_{3}\right)=\frac{1}{2} R\left(-\varepsilon_{5}\right)\left[\begin{array}{llll}
1 & 1 & 0 & 0 \\
1 & 1 & 0 & 0 \\
0 & 0 & 0 & 0 \\
0 & 0 & 0 & 0
\end{array}\right] R\left(\varepsilon_{5}\right)
$$

식 (15-20)을 식 (1)에 대입하면 체계적인 오차를 포함하는 보 다 일반적인 신호를 나타낼 수 있게 되며 뮬러 매트릭스 구 성요소와 관계가 있는 퓨리어 계수 $\left(a_{j=0,1}, \cdots 12, b_{j=1, \cdots 12}\right)$ 는 많은 연구자들에[-6,11-15] 의해 계산되고 활용되었다. 식 (7)을 선형 핏팅 계산으로 구하는 25 개의 퓨리어 계수 $\left(a_{j=0,1}, \cdots{ }_{12}, b_{j=1, \cdots 12}\right)$ 와 구하고자 하는 16 개의 뮬러 매 트릭스 구성요소, 그리고 타원편광 분석기 시스템을 구성하 는 각 광학계들의 불완전성으로 인하여 야기되는 5 가지의 체계적 오차 $\left(\delta_{1}, \theta_{1}, \delta_{2}, \theta_{2}, \theta_{3}\right)$ 는 서로 비선형적 관계가 있으며 과 평가된(overestimated) 비선형 연립방정식의 풀이 문제로 귀착된다.

다수의 분석장치에서 사용하는 일반적인 교정 방법은 샘플 을 넣지 않은 경우, 혹은 뮬러 매트릭스 구성요소가 잘 알려 진 물질 $\left(m_{i j}\right.$ 가 알려진 광학계)을 측정 공간에 설치하여 신호 를 얻고 이 측정신호를 이용하여 오차를 찾아낸 후 오차 보 정을 한 샘플의 뮬러 매트릭스 구성요소를 구해낸다. 오차 보정을 위한 일반적인 교정 방법은 두 개의 사분 파장판이 회전하는 타원 편광분석기의 경우, 측정하고자 하는 광학계 가 비어 있는 경우, 측정 물질의 뮬러 매트릭스는 단위행렬 로 나타나므로 이를 이용하여 오차 보정을 하게 된다.

타원편광분석기 구성하는 광학계의 불완전성으로 인하여 야기되는 주요한 5 가지의 체계적 오차를 포함할 경우 측정 신호를 퓨리어 변환을 통해 구한 퓨리어 계수와 측정 하고자 하는 물질 (광학계)의 뮬러 매트릭스 구성요소와의 관계는 식 (21)과 같다. 


$$
\begin{aligned}
& a_{0}=m_{11}+\left[\frac{\left(1+\cos \left(\delta_{1}\right)\right)}{4}\right] m_{12}+\left[\frac{\left(1+\cos \left(\delta_{2}\right)\right) \cos \left(2 \varepsilon_{5}\right)}{4}\right] m_{21} \\
& +\left[\frac{\left(1+\cos \left(\delta_{1}\right)\right)\left(1+\cos \left(\delta_{2}\right)\right) \cos \left(2 \varepsilon_{5}\right)}{8}\right] m_{22}+\left[\frac{\left(1+\cos \left(\delta_{2}\right)\right) \sin \left(2 \varepsilon_{5}\right)}{4}\right] m_{31} \\
& +\left[\frac{\left(1+\cos \left(\delta_{1}\right)\right)\left(1+\cos \left(\delta_{2}\right)\right) \sin \left(2 \varepsilon_{5}\right)}{8}\right] m_{32} \\
& a_{1}=\left(\frac{\sin \left(\delta_{1}\right) \sin \left(2 \varepsilon_{3}\right)}{2}\right) m_{14}+\left[\frac{\sin \left(\delta_{1}\right)\left(\cos \left(\delta_{2}\right)+1\right) \sin \left(2 \varepsilon_{3}\right) \cos \left(2 \varepsilon_{5}\right)}{4}\right] m_{24} \\
& \quad+\left[\frac{\sin \left(\delta_{1}\right)\left(\cos \left(\delta_{2}\right)+1\right) \sin \left(2 \varepsilon_{3}\right) \sin \left(2 \varepsilon_{5}\right)}{4}\right] m_{34}
\end{aligned}
$$$$
a_{2}=\left[\frac{\left(1-\cos \left(\delta_{1}\right)\right) \cos \left(4 \varepsilon_{3}\right)}{2}\right] m_{12}+\left[\frac{\left(1-\cos \left(\delta_{1}\right)\right) \sin \left(4 \varepsilon_{3}\right)}{4}\right] m_{13}
$$$$
+\left[\frac{\left(1-\cos \left(\delta_{1}\right)\right)\left(1+\cos \left(\delta_{2}\right)\right) \cos \left(4 \varepsilon_{3}\right) \cos \left(2 \varepsilon_{5}\right)}{8}\right] m_{22}
$$$$
+\left[\frac{\left(1-\cos \left(\delta_{1}\right)\right)\left(1+\cos \left(\delta_{2}\right)\right) \sin \left(4 \varepsilon_{3}\right) \cos \left(2 \varepsilon_{5}\right)}{8}\right] m_{23}
$$$$
+\left[\frac{\left(1-\cos \left(\delta_{1}\right)\right)\left(1+\cos \left(\delta_{2}\right)\right) \cos \left(4 \varepsilon_{3}\right) \sin \left(2 \varepsilon_{5}\right)}{8}\right] m_{32}
$$$$
+\left[\frac{-\left(1-\cos \left(\delta_{1}\right)\right)\left(1+\cos \left(\delta_{2}\right)\right) \sin \left(4 \varepsilon_{3}\right) \sin \left(2 \varepsilon_{5}\right)}{8}\right] m_{33}
$$$$
\begin{aligned}
& a_{3}=\left[\frac{\left(\cos \left(\delta_{1}\right)-1\right) \sin \left(\delta_{2}\right) \sin \left(2 \varepsilon_{4}-4 \varepsilon_{3}-2 \varepsilon_{5}\right)}{8}\right] m_{42} \\
& +\left[\frac{\left(\cos \left(\delta_{1}\right)-1\right) \sin \left(\delta_{2}\right) \cos \left(2 \varepsilon_{5}-2 \varepsilon_{4}+4 \varepsilon_{3}\right)}{8}\right] m_{43}
\end{aligned}
$$$$
a_{4}=\left[\frac{-\sin \left(\delta_{1}\right) \sin \left(\delta_{2}\right) \cos \left(2 \varepsilon_{4}-2 \varepsilon_{3}-2 \varepsilon_{5}\right)}{4}\right] m_{44}
$$$$
a_{5}=\left[\frac{\sin \left(\delta_{2}\right) \sin \left(2 \varepsilon_{5}-2 \varepsilon_{4}\right)}{2}\right] m_{41}+\left[\frac{\left(\cos \left(\delta_{1}\right)+1\right) \sin \left(\delta_{2}\right) \sin \left(2 \varepsilon_{5}-2 \varepsilon_{4}\right)}{4}\right] m_{42}
$$$$
a_{6}=\left[\frac{\sin \left(\delta_{1}\right) \sin \left(\delta_{2}\right) \cos \left(2 \varepsilon_{4}+2 \varepsilon_{3}-2 \varepsilon_{5}\right)}{4}\right] m_{44}
$$$$
a_{7}=\left[\frac{\left(\cos \left(\delta_{1}\right)-1\right) \sin \left(\delta_{2}\right) \sin \left(2 \varepsilon_{4}+4 \varepsilon_{3}-2 \varepsilon_{5}\right)}{4}\right] m_{42}
$$$$
+\left[\frac{-\left(\cos \left(\delta_{1}\right)-1\right) \sin \left(\delta_{2}\right) \cos \left(2 \varepsilon_{4}+4 \varepsilon_{3}-2 \varepsilon_{5}\right)}{4}\right] m_{43}
$$$$
a_{8}=\left[\frac{\left(1-\cos \left(\delta_{1}\right)\right)\left(1-\cos \left(\delta_{2}\right)\right) \cos \left(2 \varepsilon_{4}-4 \varepsilon_{3}-2 \varepsilon_{5}\right)}{16}\right]\left(m_{22}+m_{33}\right)
$$$$
+\left[\frac{\left(1-\cos \left(\delta_{1}\right)\right)\left(1-\cos \left(\delta_{2}\right)\right) \sin \left(2 \varepsilon_{4}-4 \varepsilon_{3}-2 \varepsilon_{5}\right)}{16}\right]\left(m_{32}-m_{23}\right)
$$

$$
\begin{aligned}
& a_{9}=\left[\frac{\sin \left(\delta_{1}\right)\left(1-\cos \left(\delta_{2}\right)\right) \sin \left(2 \varepsilon_{5}-4 \varepsilon_{4}+2 \varepsilon_{3}\right)}{8}\right] m_{24} \\
& +\left[\frac{\sin \left(\delta_{1}\right)\left(1-\cos \left(\delta_{2}\right)\right) \cos \left(2 \varepsilon_{5}-4 \varepsilon_{4}+2 \varepsilon_{3}\right)}{8}\right] m_{34}
\end{aligned}
$$




$$
\begin{aligned}
& a_{10}=\left[\frac{\left(1-\cos \left(\delta_{2}\right)\right) \cos \left(4 \varepsilon_{4}-2 \varepsilon_{5}\right)}{4}\right] m_{21}+\left[\frac{\left(1+\cos \left(\delta_{1}\right)\right)\left(1-\cos \left(\delta_{2}\right)\right) \cos \left(4 \varepsilon_{4}-2 \varepsilon_{5}\right)}{8}\right] m_{22} \\
& +\left[\frac{\left(1-\cos \left(\delta_{2}\right)\right) \sin \left(2 \varepsilon_{4}-2 \varepsilon_{5}\right)}{4}\right] m_{31} \\
& +\left[\frac{\left(1+\cos \left(\delta_{1}\right)\right)\left(1-\cos \left(\delta_{2}\right)\right) \sin \left(4 \varepsilon_{4}-2 \varepsilon_{5}\right)}{8}\right] m_{32} \\
& a_{11}=\left[\frac{\sin \left(\delta_{1}\right)\left(\cos \left(\delta_{2}\right)-1\right) \sin \left(2 \varepsilon_{5}-4 \varepsilon_{4}-2 \varepsilon_{3}\right)}{8}\right] m_{24} \\
& +\left[\frac{\sin \left(\delta_{1}\right)\left(\cos \left(\delta_{2}\right)-1\right) \cos \left(2 \varepsilon_{5}-4 \varepsilon_{4}-2 \varepsilon_{3}\right)}{8}\right] m_{34} \\
& a_{12}=\left[\frac{\left(1-\cos \left(\delta_{1}\right)\right)\left(1-\cos \left(\delta_{2}\right)\right) \cos \left(4 \varepsilon_{3}+4 \varepsilon_{4}-2 \varepsilon_{5}\right)}{16}\right]\left(m_{22}-m_{33}\right) \\
& +\left[\frac{\left(1-\cos \left(\delta_{1}\right)\right)\left(1-\cos \left(\delta_{2}\right)\right) \sin \left(2 \varepsilon_{3}+4 \varepsilon_{4}-2 \varepsilon_{5}\right)}{16}\right]\left(m_{23}+m_{32}\right) \\
& b_{1}=\left[\frac{\sin \left(\delta_{1}\right) \cos \left(2 \varepsilon_{3}\right)}{2}\right] m_{14}+\left[\frac{\sin \left(\delta_{1}\right)\left(\cos \left(\delta_{2}\right)+1\right) \cos \left(2 \varepsilon_{3}\right) \cos \left(2 \varepsilon_{5}\right)}{4}\right] m_{24} \\
& +\left[\frac{\sin \left(\delta_{1}\right)\left(\cos \left(\delta_{2}\right)+1\right) \cos \left(2 \varepsilon_{3}\right) \sin \left(2 \varepsilon_{5}\right)}{4}\right] m_{34} \\
& b_{2}=\left[\frac{\left(1-\cos \left(\delta_{2}\right)\right) \sin \left(4 \varepsilon_{3}\right)}{4}\right] m_{12}+\left[\frac{\left(1-\cos \left(\delta_{1}\right)\right) \cos \left(4 \varepsilon_{3}\right)}{4}\right] m_{13} \\
& +\left[\frac{\left(1-\cos \left(\delta_{1}\right)\right)\left(1+\cos \left(\delta_{2}\right)\right) \cos \left(4 \varepsilon_{3}\right) \cos \left(2 \varepsilon_{5}\right)}{8}\right] m_{23} \\
& +\left[\frac{-\left(1-\cos \left(\delta_{1}\right)\right)\left(1+\cos \left(\delta_{2}\right)\right) \sin \left(4 \varepsilon_{3}\right) \cos \left(2 \varepsilon_{5}\right)}{8}\right] m_{22} \\
& +\left[\frac{\left(1-\cos \left(\delta_{1}\right)\right)\left(1+\cos \left(\delta_{2}\right)\right) \cos \left(4 \varepsilon_{3}\right) \sin \left(2 \varepsilon_{5}\right)}{8}\right] m_{33} \\
& +\left[\frac{-\left(1-\cos \left(\delta_{1}\right)\right)\left(1+\cos \left(\delta_{2}\right)\right) \sin \left(4 \varepsilon_{3}\right) \sin \left(2 \varepsilon_{5}\right)}{8}\right] m_{32} \\
& b_{3}=\left[\frac{\left(\cos \left(\delta_{1}\right)-1\right) \sin \left(\delta_{2}\right) \cos \left(2 \varepsilon_{5}-4 \varepsilon_{4}+4 \varepsilon_{3}\right)}{8}\right] m_{42} \\
& -\left[\frac{\left(\cos \left(\delta_{1}\right)-1\right) \sin \left(\delta_{2}\right) \sin \left(2 \varepsilon_{4}-4 \varepsilon_{3}-4 \varepsilon_{5}\right)}{8}\right] m_{43} \\
& b_{4}=\left[\frac{\sin \left(\delta_{1}\right) \sin \left(\delta_{2}\right) \sin \left(2 \varepsilon_{4}-2 \varepsilon_{3}-2 \varepsilon_{5}\right)}{4}\right] m_{44} \\
& b_{5}=\left[\frac{-\sin \left(\delta_{2}\right) \cos \left(2 \varepsilon_{4}-2 \varepsilon_{5}\right)}{2}\right] m_{41} \\
& +\left[\frac{\left.-\left(\cos \left(\delta_{1}\right)+1\right) \sin \left(\delta_{2}\right)\right) \cos \left(2 \varepsilon_{5}-2 \varepsilon_{4}\right)}{4}\right] m_{42} \\
& b_{6}=\left[\frac{\sin \left(\delta_{1}\right) \sin \left(\delta_{2}\right) \sin \left(2 \varepsilon_{5}-2 \varepsilon_{3}-2 \varepsilon_{4}\right)}{4}\right] m_{44} \\
& b_{7}=\left[\frac{\left.\left(\cos \left(\delta_{1}\right)-1\right) \sin \left(\delta_{2}\right)\right) \cos \left(4 \varepsilon_{4}+4 \varepsilon_{3}-2 \varepsilon_{5}\right)}{8}\right] m_{42} \\
& +\left[\frac{\left.\left(\cos \left(\delta_{1}\right)-1\right) \sin \left(\delta_{2}\right)\right) \sin \left(4 \varepsilon_{4}+4 \varepsilon_{3}-2 \varepsilon_{5}\right)}{8}\right] m_{43}
\end{aligned}
$$


268 한국광학회지 제 25 권 제 5 호, 2014년 10 월

$$
\begin{aligned}
& b_{8}=[-]\left(m_{22}+m_{33}\right)+[-]\left(m_{23}-m_{32}\right) \\
& b_{9}=\left[\frac{\sin \left(\delta_{1}\right)\left(\cos \left(\delta_{2}\right)-1\right) \cos \left(4 \varepsilon_{4}-2 \varepsilon_{3}-2 \varepsilon_{5}\right)}{8}\right] m_{24} \\
& +\left[\frac{\sin \left(\delta_{1}\right)\left(\cos \left(\delta_{2}\right)-1\right) \sin \left(4 \varepsilon_{4}-2 \varepsilon_{3}-2 \varepsilon_{5}\right)}{8}\right] m_{34} \\
& b_{10}=\left[\frac{\left(\cos \left(\delta_{2}\right)-1\right) \sin \left(4 \varepsilon_{4}-2 \varepsilon_{5}\right)}{4}\right] m_{21} \\
& +\left[\frac{\left(\cos \left(\delta_{1}\right)+1\right)\left(\cos \left(\delta_{2}\right)-1\right) \sin \left(4 \varepsilon_{4}-2 \varepsilon_{5}\right)}{8}\right] m_{22} \\
& +\left[\frac{\left(1-\cos \left(\delta_{2}\right)\right) \cos \left(4 \varepsilon_{4}-2 \varepsilon_{5}\right)}{4}\right] m_{31} \\
& +\left[\frac{\left(1+\cos \left(\delta_{1}\right)\right)\left(1-\cos \left(\delta_{2}\right)\right) \cos \left(4 \varepsilon_{4}-2 \varepsilon_{5}\right)}{8}\right] m_{32} \\
& b_{11}=\left[\frac{\sin \left(\delta_{1}\right)\left(1-\cos \left(\delta_{2}\right)\right) \cos \left(4 \varepsilon_{4}+2 \varepsilon_{3}-2 \varepsilon_{5}\right)}{8}\right] m_{24} \\
& +\left[\frac{\sin \left(\delta_{1}\right)\left(1-\cos \left(\delta_{2}\right)\right) \sin \left(4 \varepsilon_{4}+2 \varepsilon_{3}-2 \varepsilon_{5}\right)}{8}\right] m_{34} \\
& b_{12}=\left[\frac{\left(1-\cos \left(\delta_{1}\right)\right)\left(1-\cos \left(\delta_{2}\right)\right) \sin \left(4 \varepsilon_{4}+4 \varepsilon_{3}-2 \varepsilon_{5}\right)}{16}\right]\left(m_{22}-m_{33}\right) \\
& +\left[\frac{\left(1-\cos \left(\delta_{1}\right)\right)\left(1-\cos \left(\delta_{2}\right)\right) \cos \left(4 \varepsilon_{4}+4 \varepsilon_{3}-2 \varepsilon_{5}\right)}{16}\right]\left(m_{23}+m_{32}\right)
\end{aligned}
$$

위에서 구한 퓨리어 계수들은 측정 물질의 뮬러 매트릭스 구성요소들과 관계가 있으므로 이 결과를 이용하여 뮬러 매트릭스 구성요소를 구한 결과는 식 (22)와 같다.

$$
\begin{aligned}
& m_{11}=4 a_{0}-\frac{\left(1-\varepsilon_{1}\right)}{2} m_{12}-\frac{\left(1-\varepsilon_{2}\right)}{2} m_{21}-\frac{\left(1-\varepsilon_{1}\right)\left(1-\varepsilon_{2}\right)}{4} m_{22} \\
& -\left(1-\varepsilon_{2}\right) \varepsilon_{5} m_{31}-\frac{\left(1-\varepsilon_{1}\right)\left(1-\varepsilon_{2}\right) \varepsilon_{5}}{2} m_{32} \\
& m_{12}=\frac{\left(2 m_{32} \varepsilon_{5}+m_{22}\right)\left(\varepsilon_{2}-1\right)}{2}+\frac{8\left(a_{2}-4 \varepsilon_{3} b_{2}\right)}{\left(\varepsilon_{1}+1\right)\left(16 \varepsilon_{3}^{2}+1\right)} \\
& m_{13}=\frac{\left(2 m_{33} \varepsilon_{5}+m_{23}\right)\left(\varepsilon_{2}-1\right)}{2}+\frac{32 \varepsilon_{3} a_{2}+8 b_{2}}{\left(\varepsilon_{1}+1\right)\left(16 \varepsilon_{3}^{2}+1\right)} \\
& m_{14}=2 \frac{a_{1}-\frac{\left(1-\varepsilon_{2}\right)}{2} m_{24}-\left(1-\varepsilon_{2}\right) \varepsilon_{5} m_{34}}{\varepsilon_{3}} \\
& m_{21}=\frac{m_{22}\left(\varepsilon_{1}-1\right)}{2}+\frac{8 a_{10}+16\left(\varepsilon_{5}-2 \varepsilon_{4}\right) b_{10}}{\left(\varepsilon_{1}+1\right)\left(4 \varepsilon_{5}^{2}-16 \varepsilon_{4} \varepsilon_{5}+16 \varepsilon_{4}^{2}+1\right)}
\end{aligned}
$$




$$
\begin{aligned}
& m_{22}=A / B \\
& A=16\left[-\left(4 \varepsilon_{5}^{2}-16 \varepsilon_{4} \varepsilon_{5}+16 \varepsilon_{4}^{2}+16 \varepsilon_{3}^{2}-1\right)\left(a_{8}+a_{12}\right)\right. \\
& +16 \varepsilon_{3}\left(\varepsilon_{5}-2 \varepsilon_{4}\right)\left(a_{8}-a_{12}\right)-4 \varepsilon_{3}\left(4 \varepsilon_{5}^{2}-16 \varepsilon_{4} \varepsilon_{5}+16 \varepsilon_{4}^{2}-16 \varepsilon_{3}^{2}+1\right)\left(b_{8}+b_{12}\right) \\
& +2\left(\varepsilon_{5}-2 \varepsilon_{4}\right)\left(4 \varepsilon_{5}^{2}-16 \varepsilon_{4} \varepsilon_{5}+16 \varepsilon_{4}^{2}-16 \varepsilon_{3}^{2}-1\right)\left(b_{8}-b_{12}\right) \\
& B=\left(\varepsilon_{1}+1\right)\left(\varepsilon_{2}+1\right)\left(2 \varepsilon_{5}-4 \varepsilon_{4}-4 \varepsilon_{3}-1\right)\left(2 \varepsilon_{5}-4 \varepsilon_{4}-4 \varepsilon_{3}+1\right) \\
& \times\left(2 \varepsilon_{5}-4 \varepsilon_{4}+4 \varepsilon_{3}-1\right)\left(2 \varepsilon_{5}-4 \varepsilon_{4}+4 \varepsilon_{3}+1\right) \\
& m_{23}=C / D \\
& C=16\left[-4 \varepsilon_{3}\left(4 \varepsilon_{5}^{2}-16 \varepsilon_{4} \varepsilon_{5}+16 \varepsilon_{4}^{2}-16 \varepsilon_{3}^{2}+1\right)\left(a_{8}+a_{12}\right)\right. \\
& +2\left(\varepsilon_{5}-2 \varepsilon_{4}\right)\left(4 \varepsilon_{5}^{2}-16 \varepsilon_{4} \varepsilon_{5}+16 \varepsilon_{4}^{2}-16 \varepsilon_{3}^{2}-1\right)\left(a_{8}-a_{12}\right) \\
& -\left(4 \varepsilon_{5}^{2}-16 \varepsilon_{4} \varepsilon_{5}+16 \varepsilon_{4}^{2}+16 \varepsilon_{3}^{2}-1\right)\left(b_{8}+b_{12}\right)+16 \varepsilon_{3}\left(\varepsilon_{5}-2 \varepsilon_{4}\right)\left(b_{8}-b_{12}\right) \\
& D=\left(\varepsilon_{1}+1\right)\left(\varepsilon_{2}+1\right)\left(2 \varepsilon_{5}-4 \varepsilon_{4}-4 \varepsilon_{3}-1\right)\left(2 \varepsilon_{5}-4 \varepsilon_{4}-4 \varepsilon_{3}+1\right) \\
& \times\left(2 \varepsilon_{5}-4 \varepsilon_{4}+4 \varepsilon_{3}-1\right)\left(2 \varepsilon_{5}-4 \varepsilon_{4}+4 \varepsilon_{3}+1\right) \\
& m_{24}=\frac{8\left(b_{11}-b_{9}\right)}{\left(1+\varepsilon_{2}\right)}-\frac{8\left(2 \varepsilon_{4}-\varepsilon_{5}\right)\left(b_{9}+b_{11}\right)}{\varepsilon_{3}\left(1+\varepsilon_{2}\right)} \\
& m_{31}=\frac{m_{32}\left(\varepsilon_{1}-1\right)}{2}-\frac{16\left(\varepsilon_{5}-2 \varepsilon_{4}\right) a_{10}-8 b_{10}}{\left(1+\varepsilon_{2}\right)\left(4 \varepsilon_{5}^{2}-16 \varepsilon_{4} \varepsilon_{5}+16 \varepsilon_{4}^{2}+1\right)} \\
& m_{32}=E / F \\
& E=-16\left[2\left(\varepsilon_{5}-2 \varepsilon_{4}\right)\left(4 \varepsilon_{5}^{2}-16 \varepsilon_{4} \varepsilon_{5}+16 \varepsilon_{4}^{2}-16 \varepsilon_{3}^{2}-1\right)\left(a_{8}+a_{12}\right)\right. \\
& -4 \varepsilon_{3}\left(4 \varepsilon_{5}^{2}-16 \varepsilon_{4} \varepsilon_{5}+16 \varepsilon_{4}^{2}-16 \varepsilon_{3}^{2}+1\right)\left(a_{8}-a_{12}\right) \\
& +16 \varepsilon_{3}\left(\varepsilon_{5}-2 \varepsilon_{4}\right)\left(b_{8}+b_{12}\right)-\left(4 \varepsilon_{5}^{2}-16 \varepsilon_{4} \varepsilon_{5}+16 \varepsilon_{4}^{2}+16 \varepsilon_{3}^{2}-1\right)\left(b_{8}-b_{12}\right) \\
& F=\left(\varepsilon_{1}+1\right)\left(\varepsilon_{2}+1\right)\left(2 \varepsilon_{5}-4 \varepsilon_{4}-4 \varepsilon_{3}-1\right)\left(2 \varepsilon_{5}-4 \varepsilon_{4}-4 \varepsilon_{3}+1\right) \\
& \times\left(2 \varepsilon_{5}-4 \varepsilon_{4}+4 \varepsilon_{3}-1\right)\left(2 \varepsilon_{5}-4 \varepsilon_{4}+4 \varepsilon_{3}+1\right) \\
& m_{33}=G / H \\
& G=16\left[16 \varepsilon_{3}\left(\varepsilon_{5}-2 \varepsilon_{4}\right)\left(a_{8}+a_{12}\right)-\left(4 \varepsilon_{5}^{2}-16 \varepsilon_{4} \varepsilon_{5}+16 \varepsilon_{4}^{2}-16 \varepsilon_{3}^{2}-1\right)\left(a_{8}-a_{12}\right)\right. \\
& +2\left(\varepsilon_{5}-2 \varepsilon_{4}\right)\left(4 \varepsilon_{5}^{2}-16 \varepsilon_{4} \varepsilon_{5}+16 \varepsilon_{4}^{2}-16 \varepsilon_{3}^{2}-1\right)\left(b_{8}+b_{12}\right) \\
& -4 \varepsilon_{3}\left(4 \varepsilon_{5}^{2}-16 \varepsilon_{4} \varepsilon_{5}+16 \varepsilon_{4}^{2}-16 \varepsilon_{3}^{2}+1\right)\left(b_{8}-b_{12}\right) \\
& H=\left(\varepsilon_{1}+1\right)\left(\varepsilon_{2}+1\right)\left(2 \varepsilon_{5}-4 \varepsilon_{4}-4 \varepsilon_{3}-1\right)\left(2 \varepsilon_{5}-4 \varepsilon_{4}-4 \varepsilon_{3}+1\right) \\
& \times\left(2 \varepsilon_{5}-4 \varepsilon_{4}+4 \varepsilon_{3}-1\right)\left(2 \varepsilon_{5}-4 \varepsilon_{4}+4 \varepsilon_{3}+1\right) \\
& m_{34}=\frac{8\left(a_{9}-a_{11}\right)}{\left(1+\varepsilon_{2}\right)}-\frac{8\left(\varepsilon_{5}-2 \varepsilon_{4}\right)\left(a_{9}+a_{11}\right)}{\varepsilon_{3}\left(1+\varepsilon_{2}\right)} \\
& m_{41}=\frac{8\left(1-\varepsilon_{1}\right) \varepsilon_{3}\left(b_{3}-b_{7}\right)}{\left(1+\varepsilon_{1}\right)\left(\varepsilon_{4}-\varepsilon_{5}\right)}+\frac{4\left(1-\varepsilon_{1}\right)\left(b_{3}+b_{7}\right)}{\left(1+\varepsilon_{1}\right)}-4 b_{5} \\
& m_{42}=\frac{16 \varepsilon_{3}\left(b_{7}-b_{3}\right)}{\left(1+\varepsilon_{1}\right)\left(\varepsilon_{4}-\varepsilon_{5}\right)}-\frac{8\left(b_{3}+b_{7}\right)}{\left(1+\varepsilon_{1}\right)} \\
& m_{43}=\frac{8\left(a_{7}-a_{3}\right)}{\left(1+\varepsilon_{1}\right)}-\frac{16 \varepsilon_{3}\left(a_{3}+a_{7}\right)}{\left(1+\varepsilon_{1}\right)^{2}\left(\varepsilon_{4}-\varepsilon_{5}\right)} \\
& m_{44}=-8 a_{4}=8 a_{6}
\end{aligned}
$$


퓨리어 계수들로만 관계가 있는 뮬러 매트릭스 구성요소들 을 먼저 계산한 다음 뮬러 매트릭스 구성요소들과 다른 구성 요소들과 연관이 있는 뮬러 매트릭스 구성요소를 계산해 낸 다. 즉, 위에 나타난 뮬러 매트릭스 구성요소들은 $m_{22}, m_{23}$, $m_{24}, m_{32}, m_{33}, m_{34}, m_{41}, m_{42}, m_{43}, m_{44}$,를 먼저 구하고 그 다음 $m_{12}, m_{13}, m_{14}, m_{21}, m_{31}$ 을 구한다음 마지막으로 $m_{11}$ 을 계산해 낸다.

측정공간에 샘플이 없는 빈 공간이고 5 가지 체계적 오차가 없을 경우 뮬러 매트릭스는 $4 \times 4$ 단위행렬을 나타내지만 샘플 이 없는 경우 5 가지 체계적 오차를 포함하는 여러 가지 조합에 대한 전산 모사 결과는 Table 1-4에 나타나 있다. Table 1은 두 사분파장판의 지연오차가 없고 3 가지 정렬오차만을 포함한 경 우에 대한 전산 모사 결과이다. Table 2-4는 5가지 체계적 오차 를 포함한 경우 여러 가지 조합에 대한 전산 모사 결과이다.

위의 5 가지 체계적 오차를 포함하는 경우의 뮬러 매트릭스 구 성요소를 이용한 오차 보정방법은 참고문헌 ${ }^{[1]]}$ 에 게재되어 있다.

\section{III. 결 론}

타원편광분석기는 본 논문에서 비교분석한 5 가지 체계적 인 오차 이외에도 다른 오차 발생 요소들이 있다. 그 요소들 은 검출기 반응의 비 선형성, 광원 세기의 요동, 광학계의 입 사 광에 대한 비 수직성, 회전하는 광학계의 거칠기 정도, 다 중 반사, 회전하는 시스템의 비반복성, 동일 광학계내 투과
율의 차이 등이 있다. 전산모사를 이용해 5 가지 체계적인 오 차를 분석한 결과 Table 1 에 나타나 있듯이 검광판의 정렬오 차 $\left(\epsilon_{5}\right)$ 가 야기하는 뮬러 매트릭스 오차와 사분파장판의 정렬 오차 $\left(\epsilon_{3}, \epsilon_{4}\right)$ 가 야기하는 뮬러 매트릭스 오차를 비교하면 대 각선 요소 $\left(m_{22}\right.$ 와 $\left.m_{33}\right)$ 의 상대 오차는 $0.18 \%$ 이며 비 대각선 요소 $\left(m_{23}\right.$ 와 $\left.m_{32}\right)$ 는 2 배 차이가 남을 찾아내었다. 사분파장판 의 정렬오차가 야기하는 뮬러 매트릭스 오차크기가 비 대각 선 요소 $\left(m_{23}\right.$ 와 $\left.m_{32}\right)$ 에 크게 연관되어 있음을 알아내었다. 이 결과는 실험장치 구성시 검광판의 정렬오차보다 사분파장판 의 정렬오차를 최대한 줄이는 것이 보다 정확한 뮬러매트릭 스를 획득하게 된다는 것을 알려준다. 사분 파장판의 지연오 차와 정렬오차를 비교하면(Table 1, 2, 3참조) 뮬러 매트릭스 의 대각선요소는 지연오차가 야기하는 오차가 정렬오차가 야기하는 오차보다 크고 비대각선 요소는 정렬오차가 야기 하는 오차가 더 큼을 밝혀내었다.

두 사분파장판의 지연오차가 둘 다 있을 경우와 한 개의 사분파장판이 지연오차가 있을 경우(Table 2-4참조) 뮬러 매 트릭스의 대각선 요소 $\left(m_{22}\right.$ 와 $\left.m_{33}\right)$ 는 두 사분파장판의 지연오 차가 둘 다 있을 경우가 약 $1.75 \%$ 의 상대오차를 나타내고 비 대각선요소 $\left(m_{23}\right.$ 와 $\left.m_{32}\right)$ 는 $1.69 \%$ 의 상대오차를 나타낸다.

본 연구에서 제시한 타원 편광 분석기의 교정 절차는 정렬 오차와 지연오차를 찾아내는 데 사용 가능하다. 정렬오차와 지연오차를 찾아 피드백과정을 거치면 측정 물질의 뮬러 매 트릭스 값을 보다 정확하게 얻을 수 있다.

TABLE 1. Matrices for each possible combination of azimuthal orientation errors in three optical elements of the ellipsometry (unit:deg)

\begin{tabular}{|c|c|c|c|c|c|}
\hline$\epsilon_{3}$ & $\epsilon_{4}$ & $\epsilon_{5}$ & $\delta_{1}$ & $\delta_{2}$ & Mueller Matrix \\
\hline 0 & 0 & 0 & 90 & 90 & {$\left[\begin{array}{cccc}1.0000 & -0.0 & 0.0 & 0 \\
0.0 & 1.0000 & -0.0 & 0 \\
0 & 0.0 & 1.0000 & 0 \\
0 & 0 & 0 & 1.0000\end{array}\right]$} \\
\hline 0 & 0 & 1.0 & 90 & 90 & {$\left[\begin{array}{cccc}1.0000 & 0.0 & 0.0349 & 0 \\
0.0000 & 0.9994 & -0.0349 & 0 \\
-0.0174 & 0.0349 & 0.9994 & 0 \\
0 & 0 & 0 & 0.9994\end{array}\right]$} \\
\hline 1.0 & 0 & 0 & 90 & 90 & {$\left[\begin{array}{cccc}1.0000 & 0 & 0 & 0 \\
0.0012 & 0.9976 & -0.0698 & 0 \\
0.0349 & 0.0698 & 0.9976 & 0 \\
0 & 0 & 0 & 0.9994\end{array}\right]$} \\
\hline 0 & 1.0 & 0 & 90 & 90 & {$\left[\begin{array}{cccc}1.0000 & 0.0012 & 0.0349 & 0 \\
0.000 & 0.9976 & -0.0698 & 0 \\
-0.0 & 0.0698 & 0.9976 & 0 \\
0 & 0 & 0 & 0.9994\end{array}\right]$} \\
\hline 1.0 & 0 & 1.0 & 90 & 90 & {$\left[\begin{array}{cccc}0.9988 & 0.0024 & 0.0348 & 0 \\
0.0024 & 0.9945 & -0.1045 & 0 \\
-0.0348 & 0.1045 & 0.9945 & 0 \\
0 & 0 & 0 & 0.9988\end{array}\right]$} \\
\hline 0 & 1.0 & 1.0 & 90 & 90 & {$\left[\begin{array}{cccc}1.0000 & -0.0000 & -0.0000 & 0 \\
-0.0000 & 0.9994 & 0.0349 & 0 \\
-0.0000 & -0.0349 & 0.9994 & 0 \\
0 & 0 & 0 & 1.0000\end{array}\right]$} \\
\hline 1.0 & 1.0 & 0 & 90 & 90 & {$\left[\begin{array}{cccc}1.0012 & -0.0012 & -0.0349 & 0 \\
-0.0012 & 1.0000 & 0.0000 & 0 \\
-0.0349 & 0.0000 & 1.0000 & 0 \\
0 & 0 & 0 & 0.9988\end{array}\right]$} \\
\hline 1.0 & 1.0 & 1.0 & 90 & 90 & {$\left[\begin{array}{cccc}1.0000 & -0.0000 & 0.0000 & 0 \\
0.0000 & 0.9994 & -0.0349 & 0 \\
-0.0349 & 0.0349 & 0.9994 & 0 \\
0 & 0 & 0 & 0.9994\end{array}\right]$} \\
\hline
\end{tabular}


TABLE 2. Mueller matrices for azimuthal errors including a $1^{0}$ retardation error in the first retarders

\begin{tabular}{|c|c|c|c|c|c|}
\hline$\epsilon_{3}$ & $\epsilon_{4}$ & $\epsilon_{5}$ & $\delta_{1}$ & $\delta_{2}$ & Mueller Matrix \\
\hline 0 & 0 & 0 & 91 & 90 & {$\left[\begin{array}{cccc}1.0000 & 0.0000 & 0 & 0 \\
-0.0175 & 1.0175 & 0 & 0 \\
0 & 0 & 1.0175 & 0 \\
0 & 0 & 0 & 0.9998\end{array}\right]$} \\
\hline 0 & 0 & 1.0 & 91 & 90 & {$\left[\begin{array}{cccc}1.0000 & -0.0000 & 0.0355 & 0 \\
-0.0174 & 1.0168 & -0.0355 & 0 \\
-0.0006 & 0.0355 & 1.0168 & 0 \\
0 & 0 & 0 & 0.9992\end{array}\right]$} \\
\hline 1.0 & 0 & 0 & 91 & 90 & {$\left[\begin{array}{cccc}1.0000 & 0.0000 & 0.0000 & 0 \\
-0.0162 & 1.0150 & -0.0710 & 0 \\
-0.0355 & 0.0710 & 1.0150 & 0 \\
0 & 0 & 0 & 0.9992\end{array}\right]$} \\
\hline 0 & 1.0 & 0 & 91 & 90 & {$\left[\begin{array}{cccc}1.0000 & 0.0012 & -0.0355 & 0 \\
-0.0174 & 1.0150 & 0.0710 & 0 \\
0.0012 & -0.0710 & 1.0150 & 0 \\
0 & 0 & 0 & 0.9992\end{array}\right]$} \\
\hline 1.0 & 0 & 1.0 & 91 & 90 & {$\left[\begin{array}{cccc}0.9998 & 0.0025 & 0.0354 & 0 \\
-0.0150 & 1.0119 & -0.1064 & 0 \\
-0.0360 & 0.1064 & 1.0119 & 0 \\
0 & 0 & 0 & 0.9986\end{array}\right]$} \\
\hline 0 & 1.0 & 1.0 & 91 & 90 & {$\left[\begin{array}{cccc}1.0012 & -0.0012 & -0.0355 & 0 \\
-0.0186 & 1.0175 & 0.0 & 0 \\
-0.0343 & 0.0 & 1.0175 & 0 \\
0 & 0 & 0 & 0.9986\end{array}\right]$} \\
\hline 0 & 1.0 & 1.0 & 91 & 90 & {$\left[\begin{array}{cccc}1.0000 & 0.0000 & 0.0000 & 0 \\
-0.0174 & 1.0168 & 0.0355 & 0 \\
0.0006 & -0.0355 & 1.0168 & 0 \\
0 & 0 & 0 & 0.9998\end{array}\right]$} \\
\hline 1.0 & 1.0 & 1.0 & 91 & 90 & {$\left[\begin{array}{cccc}1.0000 & -0.0000 & 0.0000 & 0 \\
-0.0174 & 1.0168 & -0.0355 & 0 \\
-0.0349 & 0.0355 & 1.0173 & 0 \\
0 & 0 & 0 & 0.9992\end{array}\right]$} \\
\hline
\end{tabular}

Table 3. Mueller matrices for azimuthal errors including a $1^{0}$ retardation error in the second retarders

\begin{tabular}{|c|c|c|c|c|c|}
\hline$\epsilon_{3}$ & $\epsilon_{4}$ & $\epsilon_{5}$ & $\delta_{1}$ & $\delta_{2}$ & Mueller Matrix \\
\hline 0 & 0 & 0 & 91 & 90 & {$\left[\begin{array}{cccc}1.0000 & 0.0000 & 0 & 0 \\
-0.0175 & 1.0175 & 0 & 0 \\
0 & 0 & 1.0175 & 0 \\
0 & 0 & 0 & 0.9998\end{array}\right]$} \\
\hline 0 & 0 & 1.0 & 91 & 90 & {$\left[\begin{array}{cccc}1.0000 & -0.0000 & 0.0355 & 0 \\
-0.0174 & 1.0168 & -0.0355 & 0 \\
-0.0006 & 0.0355 & 1.0168 & 0 \\
0 & 0 & 0 & 0.9992\end{array}\right]$} \\
\hline 1.0 & 0 & 0 & 91 & 90 & {$\left[\begin{array}{cccc}1.0000 & 0.0000 & 0.0000 & 0 \\
-0.0162 & 1.0150 & -0.0710 & 0 \\
-0.0355 & 0.0710 & 1.0150 & 0 \\
0 & 0 & 0 & 0.9992\end{array}\right]$} \\
\hline 0 & 1.0 & 0 & 91 & 90 & {$\left[\begin{array}{cccc}1.0000 & 0.0012 & -0.0355 & 0 \\
-0.0174 & 1.0150 & 0.0710 & 0 \\
0.0012 & -0.0710 & 1.0150 & 0 \\
0 & 0 & 0 & 0.9992\end{array}\right]$} \\
\hline 1.0 & 0 & 1.0 & 91 & 90 & {$\left[\begin{array}{cccc}0.9998 & 0.0025 & 0.0354 & 0 \\
-0.0150 & 1.0119 & -0.1064 & 0 \\
-0.0360 & 0.1064 & 1.0119 & 0 \\
0 & 0 & 0 & 0.9986\end{array}\right]$} \\
\hline 0 & 1.0 & 1.0 & 91 & 90 & {$\left[\begin{array}{cccc}1.0012 & -0.0012 & -0.0355 & 0 \\
-0.0186 & 1.0175 & 0.0 & 0 \\
-0.0343 & 0.0 & 1.0175 & 0 \\
0 & 0 & 0 & 0.9986\end{array}\right]$} \\
\hline 0 & 1.0 & 1.0 & 91 & 90 & {$\left[\begin{array}{cccc}1.0000 & 0.0000 & 0.0000 & 0 \\
-0.0174 & 1.0168 & 0.0355 & 0 \\
0.0006 & -0.0355 & 1.0168 & 0 \\
0 & 0 & 0 & 0.9998\end{array}\right]$} \\
\hline 1.0 & 1.0 & 1.0 & 91 & 90 & {$\left[\begin{array}{cccc}1.0000 & -0.0000 & 0.0000 & 0 \\
-0.0174 & 1.0168 & -0.0355 & 0 \\
-0.0349 & 0.0355 & 1.0173 & 0 \\
0 & 0 & 0 & 0.9992\end{array}\right]$} \\
\hline
\end{tabular}


TABLE 4. Mueller matrices for azimuthal errors including a $1^{0}$ retardation error in both retarders

\begin{tabular}{|c|c|c|c|c|c|}
\hline$\epsilon_{3}$ & $\epsilon_{4}$ & $\epsilon_{5}$ & $\overline{\delta_{1}}$ & $\overline{\bar{\delta} \delta_{2}}$ & Mueller Matrix \\
\hline 0 & 0 & 0 & 91 & 91 & {$\left[\begin{array}{cccc}1.0003 & -0.0178 & 0 & 0 \\
-0.0178 & 1.0352 & 0 & 0 \\
0 & 0 & 1.0352 & 0 \\
0 & 0 & 0 & 0.9997\end{array}\right]$} \\
\hline 0 & 0 & 1.0 & 91 & 91 & {$\left[\begin{array}{cccc}1.0003 & -0.0177 & 0.0355 & 0 \\
-0.0177 & 1.0346 & -0.0361 & 0 \\
-0.0006 & 0.0361 & 1.0346 & 0 \\
0 & 0 & 0 & 0.9991\end{array}\right]$} \\
\hline 1.0 & 0 & 0 & 91 & 91 & {$\left[\begin{array}{cccc}1.0003 & -0.0177 & 0.0012 & 0 \\
-0.0165 & 1.0327 & -0.0722 & 0 \\
-0.0361 & 0.0722 & 1.0327 & 0 \\
0 & 0 & 0 & 0.9991\end{array}\right]$} \\
\hline 0 & 1.0 & 0 & 91 & 91 & {$\left[\begin{array}{cccc}1.0003 & -0.0165 & -0.0361 & 0 \\
-0.0177 & 1.0327 & 0.0722 & 0 \\
0.0012 & -0.0722 & 1.0327 & 0 \\
0 & 0 & 0 & 0.9991\end{array}\right]$} \\
\hline 1.0 & 0 & 1.0 & 91 & 91 & {$\left[\begin{array}{cccc}0.9990 & -0.0152 & 0.0367 & 0 \\
-0.0152 & 1.0295 & -0.1082 & 0 \\
-0.0367 & 0.1082 & 1.0295 & 0 \\
0 & 0 & 0 & 0.9985\end{array}\right]$} \\
\hline 0 & 1.0 & 1.0 & 91 & 91 & {$\left[\begin{array}{cccc}1.0003 & -0.0177 & -0.0006 & 0 \\
-0.0177 & 1.0346 & 0.0361 & 0 \\
0.0006 & -0.0361 & 1.0346 & 0 \\
0 & 0 & 0 & 0.9997\end{array}\right]$} \\
\hline 1.0 & 1.0 & 0 & 91 & 91 & {$\left[\begin{array}{cccc}1.0015 & -0.0190 & -0.0349 & 0 \\
-0.0190 & 1.0352 & 0 & 0 \\
-0.0349 & 0.0 & 1.0352 & 0 \\
0 & 0 & 0 & 0.9985\end{array}\right]$} \\
\hline 1.0 & 1.0 & 1.0 & 91 & 91 & {$\left[\begin{array}{cccc}1.0003 & -0.0177 & 0.0006 & 0 \\
-0.0177 & 1.0346 & -0.0361 & 0 \\
-0.0355 & 0.0361 & 1.0346 & 0 \\
0 & 0 & 0 & 0.9991\end{array}\right]$} \\
\hline
\end{tabular}

\section{감사의 글}

이 논문은 2012년도 한밭대학교 교내학술연구비의 지원 을 받아 수행하였다.

\section{References}

1. I. An, Ellipsometry (Hanyang Univ. Press, Korea, 2000), pp. 117-176.

2. R. M. A. Azzam, "Photopolarimetric measurement of the Mueller matrix by Fourier analysis of a single detected signal," Opt. Lett. 2, 148-150 (1978).

3. D. H. Goldstein and R. A. Chipman, "Error analysis of a Mueller matrix polarimeter,” J. Opt. Soc. Am. A 7, 693-700 (1990).

4. D. H. Goldstein, "Mueller matrix dual-rotating retarder polarimeter," Appl. Opt. 31, 6676-6683 (1992).

5. K. Ichimoto, K. Shinoda, T. Yamamoto, and J. Kiyohara, "Photopolarimatric measurement system of Mueller Matrix with dual rotating waveplates," Pub. Natl. Astron, Japan 19, 11-19 (2006).

6. P. S. Hauge, "Mueller matrix ellipsometry with imperfect componensators,” J. Opt. Soc. Am. 68, 1519-1528 (1978).

7. J. K. Choi, W. Y. Shim, G. Lee, S. Y. Kim, and S. U. Park, "Study on refractive index and thickness of human stem cells by using imaging ellipsometry," Korean J. Opt. Photon. (Hankook Kwanghak Hoeji) 20, 53-56 (2009).
8. Y. J. Seo, S. U. Park, S. M. Yang, and S. Y. Kim, “Analysis of the spectro-ellipsometric data with backside reflection from semi-transparent substrate by using a rotating polarizer Ellipsometer," Korean J. Opt. Photon. (Hankook Kwanghak Hoeji) 22, 170-178 (2011).

9. S. H. Kim, E. H. Lee, I. W. Jung, J. H. Hyun, S. Y. Lee, M. I. Kang, and J. W Kim, "Optical properties of sputtered $\mathrm{Ta}_{2} \mathrm{O}_{5}$ thin films using spectroscopic ellipsometry," J. Kor. Vac. Soc. 18, 133-140 (2009).

10. K. M. Twietmeyer and R. A. Chipman, "Optimization of Mueller matrix polarimeter in the presence of error sources," Opt. Express 16, 11589-11603 (2008).

11. S. M. Nee, "Error analysis for Mueller matrix measurement," J. Opt. Soc. Am. A 20, 1651-1657 (2003).

12. M. Dubreuil, S. Rivet, B. L. Jeune, and J. Cariou, "Systematic errors specific to a snapshot Mueller matrix polarimeter," Appl. Opt. 48, 1135-1142 (2009).

13. L. Broch, A. En Naciri, and L. Johann, "Second-order systematic errors in Mueller matrix dual rotating compensator ellipsometry," Appl. Opt. 49, 3250-3258 (2010).

14. L. Broch, A. En Naciri, and L. Johann, "Systematic errors for a Mueller matrix dual rotating compensator ellipsometer," Opt. Express 16, 8814-8824 (2008).

15. D. Kim, H. D. Cheong, and B. Kim, "Systematic error correction in dual-rotating quarter-wave plate ellipsometry using overestimated optimization method," Korean J. Opt. and Photon. (Hankook KwanghakHoeji) 25, 29-37 (2014). 